\title{
Output Feedback Control of the Linear Korteweg-de Vries Equation*
}

\author{
Swann Marx $^{1}$ and Eduardo Cerpa ${ }^{2}$
}

\begin{abstract}
This paper presents the design of an output feedback control for a linear Korteweg-de Vries equation. This design is based on the backstepping method which uses a Volterra transformation. An appropriate observer is introduced and the exponential stability of the closed-loop system is proven.
\end{abstract}

\section{INTRODUCTION}

The Korteweg-de Vries equation, introduced in 1895 by the Dutch mathematicians Diederik J. Korteweg and his student Gustav de Vries, describes approximatively the behavior of long waves in a water channel of relatively shallow depth. This nonlinear partial differential equation, described by

$$
u_{t}(t, x)+u_{x}(t, x)+u_{x x x}(t, x)+u(t, x) u_{x}(t, x)=0,
$$

has been deeply studied in the controllability sense (see [9], [4], [1] and the references therein). By considering different boundary conditions on an interval $[0, L]$ and different boundary actuators, we get control results of different nature. Roughly speaking, the system is exactly controllable when the control acts from the right endpoint $x=L$, and nullcontrollable when the control acts from the left endpoint $x=$ 0 .

In this article we focus, as a first step opening further research, on the stabilizability problem for the linear Kortewegde Vries equation with a control acting on the left Dirichlet boundary condition. The studied system can be written as follows:

$$
\left\{\begin{array}{l}
u_{t}+u_{x}+u_{x x x}=0, \\
u(t, 0)=\kappa(t), u(t, L)=0, u_{x}(t, L)=0, \\
u(0, x)=u_{0}(x),
\end{array}\right.
$$

where $\kappa$ denotes the control input and $u_{0}$ is the initial condition.

Some full state feedback controls have already been designed in the literature. Let us mention [3] where a Gramianbased method is applied in the case where the control acts on the right endpoint and [11] where the backstepping method is applied with different boundary conditions. With the same approach than the last paper, we have [2] where system (2) is considered.

However, in most cases, we have no access to the full state of the system, and it is more realistic to design an

\footnotetext{
*This work has been partially supported by FONDECYT grant 1140741 , MathAmSud COSIP, and CONICYT grant ACT-1106

${ }^{1}$ Swann Marx is a student from the Electrical Engineering Department, École Normale Supérieure de Cachan, 61 Avenue du Président Wilson, 94230 Cachan, France swann . marx@ens-cachan. fr.

${ }^{2}$ Eduardo Cerpa is with Departamento de Matemática, Universidad Técnica Federico Santa María, Avda. España 1680, Valparaíso, Chile eduardo.cerpa@usm.cl.
}

output feedback control, i.e., a feedback law depending only on some partial measurements of the state.

For linear and autonomous finite-dimension systems, a stabilizability and an observability assumptions are sufficient to ensure that the separation principle holds. In other words, if there exists a controller, which asymptotically stabilizes the origin of the system and an observer which converges asymptotically to the state system, the output feedback built from this observer and this state feedback asymptotically stabilizes the origin of the system. In a PDE framework this principle is no longer true and the stability of the closed-loop system is not guaranteed.

The basic question to state the problem is which kind of measurements are we going to consider. The case of a boundary measurement is the most challenging one and the natural choice for the $\mathrm{KdV}$ equation (2) should be $y(t)=$ $u_{x}(t, 0)$. Unfortunately, the system is not observable with this choice. In fact, if $L=2 \pi$, then the stationary solution $u(t, x)=1-\cos (x)$, satisfies (2) with $\kappa(t)=0$ and in addition $u_{x}(t, 2 \pi)=0$ for any time $t$. The length $2 \pi$ is not the only one for which observability does not hold (see e.g. [8], [1]).

In this paper we consider the output given by

$$
y(t)=u_{x x}(t, L) .
$$

By using this measurement, we build an observer and apply the backstepping method to design an output feedback control which exponentially stabilizes the closed-loop system. The trace $u_{x x}(t, L)$ can be considered itself as a boundary condition or even a control ([5], [4]) and therefore the choice of this output is not an artificial one.

This paper is organized as follows. In Section II, we state our main result. Section III is devoted to recall the state feedback control designed in [2]. In IV, we state some regularity results needed to consider the measurement (3) as a continuous function. The observer is built in Section V. In Section VI, the stability of the closed loop controllerobserver system is proven. Finally, Section VII states some conclusions.

\section{MAin Result}

Based on [7] and [10], we construct the following observer:

$$
\left\{\begin{array}{l}
\hat{u}_{t}+\hat{u}_{x}+\hat{u}_{x x x}+p_{1}(x)\left[y(t)-\hat{u}_{x x}(t, L)\right]=0, \\
\hat{u}(t, 0)=\kappa(t), \hat{u}(t, L)=\hat{u}_{x}(t, L)=0, \\
\hat{u}(0, x)=0 .
\end{array}\right.
$$

Theorem 1. For any $\lambda>0$, there exist a feedback law $\kappa(t):=\kappa(\hat{u}(t, x))$, a function $p_{1}=p_{1}(x)$, and a constant 
$C>0$ such that the coupled system (2)-(3)-(4) is globally exponentially stable with a decay rate equals to $\lambda$, i.e., for any $u_{0} \in H^{3}(0, L)$ we have

$$
\|u(t, \cdot)\|_{H^{3}(0, L)}+\|\hat{u}(t, \cdot)\|_{L^{2}(0, L)} \leq C e^{-\lambda t}\left\|u_{0}\right\|_{H^{3}(0, L)}
$$

\section{Full STATE FeEdback Design}

In [2] the following Volterra transformation is introduced

$$
w(x)=\Pi(u(x)):=u(x)-\int_{x}^{L} k(x, y) u(y) d y .
$$

The function $k$ is chosen such that the trajectory $u=u(t, x)$, solution of (2), with control

$$
\kappa(t)=\int_{0}^{L} k(0, y) u(t, y) d y
$$

is mapped into the trajectory $w=w(t, x)$, solution of the linear system

$$
\left\{\begin{array}{l}
w_{t}+w_{x}+w_{x x x}+\lambda w=0, \\
w(t, 0)=w(t, L)=w_{x}(t, L)=0,
\end{array}\right.
$$

which is exponentially stable if $\lambda>0$. In fact, from a Lyapunov approach, we get

$$
\begin{aligned}
\frac{d}{d t} \int_{0}^{L}|w(t, x)|^{2} d x & =-\left|w_{x}(t, 0)\right|^{2}-2 \lambda \int_{0}^{L}|w(t, x)|^{2} d x \\
& \leq-2 \lambda \int_{0}^{L}|w(t, x)|^{2} d x,
\end{aligned}
$$

which gives an exponential decay rate equals to $\lambda$ for the $L^{2}$-norm of the state $w$.

The kernel function $k=k(x, y)$ is characterized by:

$$
\left\{\begin{array}{l}
k_{x x x}+k_{y y y}+k_{x}+k_{y}=-\lambda k, \text { in } \mathcal{T} \\
k(x, L)=0, \text { in }[0, L], \\
k(x, x)=0, \text { in }[0, L], \\
k_{x}(x, x)=\frac{\lambda}{3}(L-x), \text { in }[0, L]
\end{array}\right.
$$

where $\mathcal{T}:=\{(x, y) / x \in[0, L], y \in[x, L]\}$. The solution of (10) exist. This is proved by using the method of successive approximations. Unlikely the case of heat or wave equations, we do not have an explicit solution.

In [2] it is proved that the transformation (6) linking (2) and (8) is invertible, continuous and its inverse is also continuous. Therefore, the exponential decay for $w$, solution of (8), implies the exponential decay for the solution $u$ controlled by (7). Thus, with this method, the following theorem is proven.

Theorem 2. (State feedback stabilization for $K d V$ ([2])

For any $\lambda>0$, there exist a feedback control law $\kappa=$ $\kappa(u(t,)$.$) and C>0$ such that

$$
\|u(t, .)\|_{L^{2}(0, L)} \leq C e^{-\lambda t}\left\|u_{0}\right\|_{L^{2}(0, L)}
$$

for any solution of (2)-(7).

\section{REGULARITY RESUlT}

As we said in the introduction, we consider

$$
y(t)=u_{x x}(t, L)
$$

as a partial measurement of the solution. However, since we have the trace of the second derivative with respect to $x$ of $u$, we need a regularity stronger than in [2]. Indeed, we ask the output $y(t)$ to be a continuous function. Thus we have the following lemma.

Lemma 1. Let us consider system

$$
\left\{\begin{array}{l}
u_{t}+u_{x}+u_{x x x}=0, \\
u(t, 0)=\kappa(t), u(t, L)=0, u_{x}(t, L)=0, \\
u(0, x)=u_{0}(x),
\end{array}\right.
$$

where $u_{0} \in H^{3}(0, L)$ and $\kappa(t) \in H^{1}(0, T)$. Then $u \in$ $C\left([0, T], H^{3}(0, L)\right) \cap L^{2}\left(0, T ; H^{4}(0, L)\right)$ and $u_{x x}(\cdot, L) \in$ $C([0, T])$.

Proof. This proof is based on [6]. Let us consider the following coordinates transformation

$$
v=u_{t} \text {. }
$$

The dynamics of $v$ can be written as follows:

$$
\left\{\begin{array}{l}
v_{t}+v_{x x x}+v_{x}=0 \\
v(t, 0)=\dot{\kappa}(t) \in L^{2}(0, T), v(t, L)=v_{x}(t, L)=0, \\
v(0, x)=\left(-u_{0}^{\prime \prime \prime}-u_{0}^{\prime}\right) \in L^{2}(0, L) .
\end{array}\right.
$$

By already known well-posedness results for $\mathrm{KdV}$ ([6]), we get

$$
\begin{aligned}
& v \in C\left([0, T], L^{2}(0, L)\right) \cap L^{2}\left(0, T ; H^{1}(0, L)\right) \\
\Rightarrow & u_{t} \in C\left([0, T], L^{2}(0, L)\right) \cap L^{2}\left(0, T ; H^{1}(0, L)\right) \\
\Rightarrow & u \in C\left([0, T], H^{3}(0, L)\right) \cap L^{2}\left(0, T ; H^{4}(0, L)\right) \\
\Rightarrow & u_{x x} \in C\left([0, T], H^{1}(0, L)\right) \cap L^{2}\left(0, T ; H^{2}(0, L)\right) \\
\Rightarrow & u_{x x} \in C([0, T] \times[0, L]) \\
\Rightarrow & u_{x x}(\cdot, L) \in C([0, T])
\end{aligned}
$$

Thus it concludes the proof of Lemma 1.

\section{OBSERVER DESIGN}

Based on [7], and more precisely on [10], we can write, for system

$$
\left\{\begin{array}{l}
u_{t}+u_{x}+u_{x x x}=0 \\
u(t, 0)=\kappa(t), u(t, L)=u_{x}(t, L)=0 \\
y(t)=u_{x x}(t, L)
\end{array}\right.
$$

the corresponding observer

$$
\left\{\begin{array}{l}
\hat{u}_{t}+\hat{u}_{x}+\hat{u}_{x x x}+p_{1}(x)\left[u_{x x}(t, L)-\hat{u}_{x x}(t, L)\right]=0 \\
\hat{u}(t, 0)=\kappa(t), \hat{u}(t, L)=\hat{u}_{x}(t, L)=0
\end{array}\right.
$$

The construction of the observer is based on the finitedimensional design for $\dot{x}=A x+B u, y=C x$, which proposes the observer $\dot{\hat{x}}=A \hat{x}+B u+L(y-C \hat{x})$. If we consider the error $e=x-\hat{x}$, then $\dot{e}=(A-L C) e$, 
and we have to look for a matrix $L$ insuring a good performance. Because of the infinite-dimensional framework we are working in, a matrix $L$ is not enough. Thus we need a function $p_{1}(x)$.

In our case, we consider the error $\tilde{u}:=u-\hat{u}$, which satisfies

$$
\left\{\begin{array}{l}
\tilde{u}_{t}+\tilde{u}_{x}+\tilde{u}_{x x x}-p_{1}(x) \tilde{u}_{x x}(t, L)=0, \\
\tilde{u}(t, 0)=\tilde{u}(t, L)=\tilde{u}_{x}(t, L)=0 .
\end{array}\right.
$$

Given a positive parameter $\lambda$, we look for a transformation $\Pi_{o}$ defined by

$$
\tilde{u}(x)=\Pi_{o}(\tilde{w}(x))=\tilde{w}(x)-\int_{x}^{L} p(x, y) \tilde{w}(y) d y
$$

such that the trajectory $\tilde{u}$, solution of (19) is mapped into the trajectory $\tilde{w}=\tilde{w}(t, x)$, solution of the linear system

$$
\left\{\begin{array}{l}
\tilde{w}_{t}+\tilde{w}_{x}+\tilde{w}_{x x x}+\lambda \tilde{w}=0 \\
\tilde{w}(t, 0)=0, \tilde{w}(t, L)=0, \tilde{w}_{x}(t, L)=0,
\end{array}\right.
$$

which is exponentially stable with a decay rate depending on the value of $\lambda$ as shown in (9).

Now, the key step is to find the kernel $p=p(x, y)$ such that $\tilde{u}(t, x)=\Pi_{o}(\tilde{w}(t, x))$ satisfies (19). By focusing on (20) and using the Leibniz rules, we get:

- Differentiation along (21)

$\tilde{u}_{t}=\tilde{w}_{t}(x)-\int_{x}^{L} p(x, y)\left[-\tilde{w}_{y}(y, t)-\tilde{w}_{y y y}(y, t)-\lambda \tilde{w}(y, t)\right] d y$

$$
\begin{aligned}
& =\tilde{w}_{t}(x)-\int_{x}^{L}\left(-\lambda p(x, y)+p_{y}(x, y)+p_{y y y}(x, y)\right) \tilde{w}(y, t) d y \\
& +p(x, L) \tilde{w}(L, t)-p(x, x) \tilde{w}(x, t)+p(x, L) \tilde{w}_{x x}(L, t) \\
& -p(x, x) \tilde{w}_{x x}(x, t)+p_{y}(x, x) \tilde{w}_{x}(x)-p_{y}(x, L) \tilde{w}_{x}(L, t) \\
& +p_{y y}(x, L) \tilde{w}(L, t)-p_{y y}(x, x) \tilde{w}(x, t)
\end{aligned}
$$

- Three differentations with respect to the variable $x$

$$
\begin{aligned}
\tilde{u}_{x}(x, t) & =\tilde{w}_{x}(x, t)+p(x, x) \tilde{w}(x, t)-\int_{x}^{L} p_{x}(x, y) \tilde{w}(t, y) d y \\
\tilde{u}_{x x}(t, x) & =\tilde{w}_{x x}(t, x)+\frac{d}{d x} p(x, x) \tilde{w}(t, x)+p(x, x) \tilde{w}_{x}(t, x) \\
& +p_{x}(x, x) \tilde{w}(t, x)-\int_{x}^{L} p_{x x}(x, y) \tilde{w}(t, y) d y
\end{aligned}
$$

$$
\begin{aligned}
\tilde{u}_{x x x}(t, x) & =\tilde{w}_{x x x}(t, x)+\frac{d^{2}}{d x^{2}} p(x, x) \tilde{w}(t, x) \\
& +2 \frac{d}{d x} p(x, x) \tilde{w}_{x}(t, x) \\
& +p(x, x) \tilde{w}_{x x}(t, x)+\frac{d}{d x} p_{x}(x, x) \tilde{w}(t, x) \\
& +p_{x}(x, x) \tilde{w}_{x}(t, x)+p_{x x}(x, x) \tilde{w}(t, x) \\
& -\int_{x}^{L} p_{x x x}(x, y) \tilde{w}(t, x) d y
\end{aligned}
$$

By adding (22), (23) and (25), we get

$$
\begin{aligned}
& \tilde{u}_{t}+\tilde{u}_{x}+\tilde{u}_{x x x}-p_{1}(x) \tilde{u}_{x x}(L)= \\
& \tilde{w}_{t}(t, x)+\tilde{w}_{x}(t, x)+\tilde{w}_{x x x}(t, x)+\lambda \tilde{w}(t, x) \\
& -\int_{x}^{L}\left(-\lambda p(x, y)+p_{y}(x, y)+p_{y y y}(x, y)\right. \\
& \left.+p_{x x x}(x, y)+p_{x}(x, y)\right) \tilde{w}(y, t) d y \\
& +\tilde{w}_{x}(t, x)\left(2 \frac{d}{d x} p(x, x)+p_{x}(x, x)+p_{y}(x, x)\right) \\
& +\tilde{w}(t, x)\left(p_{x x}(x, x)+\frac{d^{2}}{d x^{2}} p(x, x)+\frac{d}{d x} p_{x}(x, x)\right. \\
& \left.-p_{y y}(x, x)-\lambda\right)+\left(p(x, L)-p_{1}(x)\right) \tilde{w}_{x x}(L, t) \\
& +p(x, L) \tilde{w}(L, t)-p_{y}(x, L) \tilde{w}_{x}(L, t) .
\end{aligned}
$$

From this equation, we get four conditions:

1. Equation for $(x, y) \in \mathcal{T}$ :

$$
p_{y y y}+p_{x x x}+p_{y}+p_{x}=\lambda p .
$$

2. First boundary condition on $(x, x)$ for $x \in[0, L]$ :

$$
2 \frac{d}{d x} p(x, x)+p_{x}(x, x)+p_{y}(x, x)=0 .
$$

3. Second boundary condition on $(x, x)$ for $x \in[0, L]$ :

$$
\begin{aligned}
& \frac{d^{2}}{d x^{2}} p(x, x)+\frac{d}{d x} p_{x}(x, x) \\
& +p_{x x}(x, x)-p_{y y}(x, x)-\lambda=0 .
\end{aligned}
$$

4. Appropriate choice of $p_{1}$ :

$$
p(x, L)=p_{1}(x) .
$$

Recall that $\mathcal{T}:=\{(x, y) / x \in[0, L], y \in[x, L]\}$.

Moreover, note also that, by setting $x=0$ in (20), we get:

$$
p(0, y)=0, \quad \forall y \in[0, L] .
$$

In addition, we have

$$
\tilde{w}(t, L)=\tilde{u}(t, L)=\tilde{w}_{x}(t, L)=0 .
$$

Finally, the kernel $p$ satisfies the following PDE:

$$
\left\{\begin{array}{l}
p_{x x x}(x, y)+p_{y y y}(x, y) \\
+p_{y}(x, y)+p_{x}(x, y)=\lambda p(x, y), \quad(x, y) \in \mathcal{T}, \\
p(x, x)=0, \quad x \in[0, L], \\
p_{x}(x, x)=\frac{\lambda}{3} x, \quad x \in[0, L], \\
p(0, y)=0, \quad y \in[0, L] .
\end{array}\right.
$$

Let us make the following change of variable:

$$
\bar{x}=L-y, \quad \bar{y}=L-x,
$$

and define $F(\bar{x}, \bar{y}):=p(x, y)$. Hence:

$$
\left\{\begin{array}{l}
F_{\bar{x} \bar{x} \bar{x}}(\bar{x}, \bar{y})+F_{\bar{y} \bar{y} \bar{y}}(\bar{x}, \bar{y}) \\
+F_{\bar{y}}(\bar{x}, \bar{y})+F_{\bar{x}}(\bar{x}, \bar{y})=-\lambda F(\bar{x}, \bar{y}) \quad(\bar{x}, \bar{y}) \in \mathcal{T} \\
F(\bar{x}, \bar{x})=0 \quad \bar{x} \in[0, L] \\
F_{\bar{x}}(\bar{x}, \bar{x})=\frac{\lambda}{3}(L-\bar{x}) \quad \bar{x} \in[0, L] \\
F(\bar{x}, L)=0 \quad \bar{y} \in[0, L]
\end{array}\right.
$$


This PDE has already been studied in [2], where no explicit solution has been found, but where the existence of a solution has been proved. Hence, we can conclude that the kernel $p:=p(x, y)$ exists. Note that the function $\Pi_{o}$ defined by (20) is linear (by definition) and continuous (because of the existence of $p$ ).

\section{STABILITY ANALYSIS OF THE CLOSED LOOP SYSTEM}

Instead of dealing directly with the controlled state $u$ and the observer state $\hat{u}$, we consider the evolution of the couple $(\tilde{u}, \hat{u})$ where $\tilde{u}$ stands for the error $\tilde{u}=u-\hat{u}$, as introduced in Section V.

By using the output feedback control

$$
\kappa(t)=\int_{0}^{L} k(0, y) \hat{u}(t, y) d y,
$$

the transformation $\Pi$ defined in (6) and its inverse, and the transformation $\Pi_{o}$ defined in (20) and its inverse, we can see that $(\tilde{u}, \hat{u})$ are mapped into $(\tilde{w}, \hat{w})=\left(\Pi_{o}^{-1}(\tilde{u}), \Pi(\hat{u})\right)$ solutions of the target system

$$
\left\{\begin{array}{l}
\hat{w}_{t}+\hat{w}_{x}+\hat{w}_{x x x}+\lambda \hat{w}= \\
-\left\{p_{1}(x)-\int_{x}^{L} k(x, y) p_{1}(y) d y\right\} \tilde{w}_{x x}(t, L), \\
\hat{w}(0)=\hat{w}(L)=\hat{w}_{x}(L)=0 \\
\tilde{w}_{t}+\tilde{w}_{x}+\tilde{w}_{x x x}+\lambda \tilde{w}=0 \\
\tilde{w}_{x}(0)=\tilde{w}(L)=\tilde{w}_{x}(L)=0 .
\end{array}\right.
$$

Note that the parameter $\lambda$ is the same for the observer and the system itself.

Given that $\Pi, \Pi_{o}$ are continuous maps, invertible and their inverse maps are also continuous, the exponential stability of (36) would imply the exponential stability of the closed loop system and therefore the proof of Theorem 1 will be ended.

In order to prove the exponential stability of (36), we use a Lyapunov argument. Let us consider the following function,

$$
V(t)=V_{1}(t)+V_{2}(t)+V_{3}(t)
$$

where

$$
\begin{aligned}
& V_{1}(t)=\frac{A}{2} \int_{0}^{L}|\hat{w}(t, x)|^{2} d x \\
& V_{2}(t)=\frac{B}{2} \int_{0}^{L}|\tilde{w}(t, x)|^{2} d x \\
& V_{3}(t)=\frac{B}{2} \int_{0}^{L}\left|\tilde{w}_{t}(t, x)\right|^{2} d x
\end{aligned}
$$

with $A, B$ to be chosen later.

Remark 1. We can prove that this Lyapunov function is equivalent to the one obtained by replacing $V_{3}(t)$ by

$$
\tilde{V}_{3}(t)=\frac{B}{2} \int_{0}^{L}\left|\tilde{w}_{x x x}(t, x)\right|^{2} d x
$$

By this, we mean that the exponential decay of one of them implies the exponential decay of the other one. In fact, we can prove that there exist positive constants $d_{1}, d_{2}$ such that

$$
d_{1}\left(V_{2}(t)+\tilde{V}_{3}(t)\right) \leq V_{2}(t)+V_{3}(t) \leq d_{2}\left(V_{2}(t)+\tilde{V}_{3}(t)\right) .
$$

Taking the time derivative of the function $V(t)$, we get after some computations that

$$
\begin{aligned}
\dot{V}_{1}(t)= & A \int_{0}^{L} \hat{w}_{t}(t, x) \hat{w}(t, x) d x \\
& \leq\left(-A \lambda+D^{2}\right) \int_{0}^{L}|\hat{w}(t, x)|^{2} d x+A^{2}\left|\tilde{w}_{x x}(t, L)\right|^{2} \\
& =2\left(-\lambda+\frac{D^{2}}{A}\right) V_{1}(t)+A^{2}\left|\tilde{w}_{x x}(t, L)\right|^{2}
\end{aligned}
$$

where $D:=\max _{x \in[0, L]}\left\{p_{1}(x)-\int_{x}^{L} k(x, y) p_{1}(y) d y\right\}$.

From the same computation as in (9), we see that

$$
\dot{V}_{2}(t) \leq-2 \lambda V_{2}(t) \text {. }
$$

Moreover, thanks to the regularity $H^{3}(0, L)$, the same computation can be applied to $z=\tilde{w}_{t}$ (see the proof of Lemma 1) to obtain

$$
\dot{V}_{3}(t) \leq-2 \lambda V_{3}(t)
$$

Thus, we get:

$$
\begin{aligned}
\dot{V}(t) \leq & 2\left(-\lambda+\frac{D^{2}}{A}\right) V_{1}(t)+A^{2}\left|\tilde{w}_{x x}(t, L)\right|^{2} \\
& -2 \lambda V_{2}(t)-2 \lambda V_{3}(t) .
\end{aligned}
$$

We need to find an upper bound for $\left|\tilde{w}_{x x}(t, L)\right|^{2}$. We multiply

$$
\left\{\begin{array}{l}
\tilde{w}_{t}+\tilde{w}_{x}+\tilde{w}_{x x x}+\lambda \tilde{w}=0, \\
\tilde{w}(0)=\tilde{w}(L)=\tilde{w}_{x}(L)=0,
\end{array}\right.
$$

by $x \tilde{w}_{x x}$ and after some computations we get

$$
\begin{aligned}
\left|\tilde{w}_{x x}(t, L)\right|^{2} \leq & \left(\frac{1}{L}+L\right)\left\|\tilde{w}_{x x}\right\|_{L^{2}(0, L)}^{2} \\
& +\left(2 \lambda+\frac{1}{L}\right)\left\|\tilde{w}_{x}\right\|_{L^{2}(0, L)}^{2} \\
& +\frac{1}{L}\left\|\tilde{w}_{t}\right\|_{L^{2}(0, L)}^{2}
\end{aligned}
$$

and finally the existence of $a, b>0$ such that

$$
\left|\tilde{w}_{x x}(t, L)\right|^{2} \leq a\|\tilde{w}\|_{L^{2}(0, L)}^{2}+b\left\|\tilde{w}_{t}\right\|_{L^{2}(0, L)}^{2}
$$

Remark 2. Here, we have used that the norm $\|f\|_{H^{3}(0, L)}$ and the norm $\|f\|_{L^{2}(0, L)}+\left\|f_{x x x}\right\|_{L^{2}(0, L)}$ are equivalent. See also Remark 1.

We use the latter inequality to write:

$$
\begin{aligned}
\dot{V}(t) \leq & 2\left(-\lambda+\frac{D^{2}}{A}\right) V_{1}(t)+2 a \frac{A^{2}}{B} V_{2}(t) \\
& +2 b \frac{A^{2}}{B} V_{3}(t)-2 \lambda V_{2}(t)-2 \lambda V_{3}(t) .
\end{aligned}
$$

Therefore,

$$
\begin{aligned}
\dot{V}(t) \leq & 2\left(-\lambda+\frac{D^{2}}{A}\right) V_{1}(t)+2\left(-\lambda+\frac{a A^{2}}{B}\right) V_{2}(t) \\
& 2\left(-\lambda+\frac{b A^{2}}{B}\right) V_{3}(t) .
\end{aligned}
$$


In this way, by tuning $A, B$ large enough, we get for any $\varepsilon>0$ that

$$
\dot{V}(t) \leq 2(-\lambda+\varepsilon) V(t)
$$

which gives an exponential stability with decay rate as close to $\lambda$ as we want. The rapid stabilization is achieved because the parameter $\lambda$ can be chosen as large as desired.

It concludes the proof of the stability of the closed loop system with the output feedback control law depending on a boundary measurement of the state.

\section{CONCLUSIONS}

In this paper we have designed an output feedback controller for the linear Korteweg-de Vries equation posed on a bounded interval. This boundary feedback control acts on the Dirichlet boundary condition on the left endpoint and exponentially stabilizes the system by using the boundary measurement $y(t)=u_{x x}(t, L)$. Because of this choice of $y(t)$, we have to work on a more regular framework, given by $H^{3}(0, L)$ as the state space. The backstepping method is applied together a classical observer in order to build the control.

This work is the first one addressing the output feedback problem for the Korteweg-de Vries equation and opens several possible extensions. Different location for the control or different boundary conditions can be considered as in [2], [11]. The choice of the measurement is particularly interesting. For instance, it would be nice to deal with the collocated case, which could be harder than the noncollocated problem considered in this paper. Of course, the nonlinear case also appears as a natural next step in the study of this control system. In that context, even performing simulations of the closed loop system may be an interesting challenge.

\section{REFERENCES}

[1] E. Cerpa. Control of a Korteweg-de Vries equation: a tutorial. Mathematical Control and Related Fields, 4(1):45-99, 2014.

[2] E. Cerpa and J.-M. Coron. Rapid stabilization for a Korteweg-de Vries equation from the left Dirichlet boundary condition. IEEE Trans. Automat. Control, 58(7):1688-1695, 2013.

[3] E. Cerpa and E. Crépeau. Rapid exponential stabilization for a linear Korteweg-de Vries equation. Discrete Contin. Dyn. Syst. Ser. B, 11(3):655-668, 2009.

[4] E. Cerpa, I. Rivas, and B.-Y. Zhang. Boundary controllability of the Korteweg-de Vries equation on a bounded domain. SIAM J. Control Optim., 51(4):2976-3010, 2013.

[5] T. Colin and J.-M. Ghidaglia. An initial-boundary-value problem for the Korteweg-de Vries equation posed on a finite interval. $A d v$. Differential Equations, 6:1463-1492, 2001.

[6] O. Glass and S. Guerrero. Some exact controllability results for the linear kdv equation and uniform controllability in the zero-dispersion limit. Asymptotic Analysis, 60(1):61-100, 2008.

[7] M. Krstic and A. Smyshlyaev. Boundary Control of PDEs: A Course on Backstepping Designs. SIAM, 2008.

[8] L. Rosier. Exact boundary controllability for the Korteweg-de Vries equation on a bounded domain. ESAIM: Control, Optimisation and Calculus of Variations, 2:33-55, 1997.

[9] L. Rosier and B.-Y. Zhang. Control and stabilization of the Kortewegde Vries equation: recent progresses. J. Syst. Sci. Complex., 22(4):647682, 2009.

[10] A. Smyshlyaev and M. Krstic. Backstepping observers for a class of parabolic PDEs. Systems \& Control letters, 54(7):613-625, 2005.
[11] S. Tang and M. Krstic. Stabilization of Linearized Korteweg-de Vries Systems with Anti-diffusion. In American Control Conference (ACC), pages 3302-3307, 2013. 